\title{
Semi-Automatic Global Contrast Enhancement
}

\author{
S. Somorjeet Singh \\ Department of Computer Science \\ Manipur University, Canchipur
}

\begin{abstract}
Since local contrast enhancement is not sufficient for a detailed visibility of an image, global enhancement also cannot be neglected. Among the global contrast enhancement methods, the automatic enhancement method like Global Histogram Equalization is not always desirable to utilize it for some images that some portions are overexposure and some portions are underexposure and no user's choice is available. And for other global enhancement methods, when the number of user defined parameters are more, more number of different choices are available and the enhancement level can be adjusted more accurately. However, the user's convenience is less when more number of user defined parameters. For the sake of user's convenience, the semi-automatic contrast enhancement method using single user defined parameter works better for some images.
\end{abstract}

\section{Keywords}

Global Contrast Enhancement, Semi-Automatic, Single Parameter Control.

\section{INTRODUCTION}

Good quality images are always desired by everyone, because the objects in the good quality images are clear and quite perceptible to everyone. Sometimes, someone may have a very rare and valuable image in low quality because the visual quality of an image depends on various factors like recording, recording device, light incident on the objects and processing etc. There are several low quality image types, and low contrast image is one of the low quality image types. Low contrast images are those whose intensity levels of the pixels are in narrow range. The visual quality of such type of images can be improved using different enhancement techniques in different domain like spatial domain, frequency domain etc. Several image enhancement techniques have been introduced and developed by different authors.

Linear contrast stretching and histogram equalization are two widely utilized methods for global image enhancement [1]-[5]. Although these methods are simple, they do not define local details. The enhancement techniques based on local contrast are necessary because the image characteristics differ considerably from one region to another in the same image.

There are several local contrast enhancement methods. The adaptation of histogram stretching method over a neighbourhood around the candidate pixel was used by Dorst [6] for local contrast stretching, then it was followed by several modifications [7]-[9] of histogram equalization based on adapting the same over a sub-region of the image. Lee [10], [11] suggested a local contrast stretching method that makes use of local statistics of a predefined neighbourhood in modifying the gray level of a pixel. Another local contrast enhancement method was designed by Narendra and Fitch [12] so that the amplification factor too to be a function of the pixel based on the local gray level statistics over the same neighbourhood in which contrast gain is inversely proportional to Local Standard Deviation (LSD). However, Dah-Chung [13] observed that image enhancement with contrast gain which is constant or inversely proportional to the LSD produces either ringing artifacts or noise over enhancement due to the use of too large contrast gains in regions with high and low activities and developed a new method in which gain is a non-linear function of LSD. A multi-window extension of the technique that the contrast gain is inversely proportional to the LSD was introduced by Schutte [14] and showed how the window sizes should be chosen. An improved multi-window real-time high frequency enhancement scheme based on LSD in which gain is a non-linear function of the detail energy was implemented by Sascha [15]. However, some enhancement methods which are using the LSD suffer from divide by zero conditions when LSD's of some pixels of the input image are having the value zero. This divide by zero condition can be overcome [16] by modifying the LSD's, changing with a very small negligible value.

Although the local contrast enhancements improved the quality of the low contrast images, there is still a room needed for global contrast enhancement because the image enhanced by local contrast methods may be still poor in global contrast for some images.

This paper is organized as follows: Section 2 briefly reviews some global contrast enhancement methods. Section 3 describes the proposed method. Section 4 shows experimental results and discussion. And Section 5 is for conclusion.

\section{REVIEW ON SOME GLOBAL CONTRAST ENHANCEMENT METHODS}

Some global contrast enhancement methods are reviewed in this section.

First, among the automatic enhancement methods, Global Histogram Equalization (GHE) is one of the most common global contrast enhancement methods. Here, the histogram equalization transformation for the interval $[0, \mathrm{~L}-1]$ can be defined [2] as

$S_{k}=T\left(r_{k}\right)=(L-1) \sum_{j=0}^{k} p_{r}\left(r_{j}\right)=\frac{(L-1)}{M N} \sum_{j=0}^{k} n_{j}$

where $\boldsymbol{k}=\mathbf{0}, \mathbf{1}, 2, \ldots, \boldsymbol{L}-\mathbf{1}, \boldsymbol{r}_{\boldsymbol{k}}$ is a pixel in the input image, $\boldsymbol{S}_{\boldsymbol{k}}$ is the corresponding pixel in the output image, $\boldsymbol{p}_{\boldsymbol{r}}\left(\boldsymbol{r}_{j}\right)$ is the Probability Density Function of $\boldsymbol{r}_{j}, \boldsymbol{M N}$ is the total number of pixels in the image and $\boldsymbol{n}_{\boldsymbol{j}}$ is the number of pixels that have intensity $\boldsymbol{r}_{\boldsymbol{j}}$.

Although GHE is one of the most frequently used enhancement methods, it is not always desirable [17] because over enhancement is occurred in some parts of the output image and some parts of the output image are under exposure, i.e. some information are defined. There is no user's option to control the enhancement level to employ this method.

Next is one of the methods whose enhancement is controlled by two user defined parameters. As a simple implementation, the following method [1] can be employed using two user defined parameters: 
$F(x, y)=m * f(x, y)-n$

(2)

where $\boldsymbol{F}(\boldsymbol{x}, \boldsymbol{y})=0$ if $(\boldsymbol{m} * f(x, y)-n)<0$, or $\boldsymbol{F}(\boldsymbol{x}, \boldsymbol{y})=1$ if $(\boldsymbol{m} * f(x, y)$ $\boldsymbol{n})>\boldsymbol{1}, \boldsymbol{F}(\boldsymbol{x}, \boldsymbol{y})$ is the output image, $\boldsymbol{f}(\boldsymbol{x}, \boldsymbol{y})$ is the input image, $\boldsymbol{m}$ is a global multiplier and $\mathrm{n}$ is a real value such that $\boldsymbol{m}>\mathbf{0}, \boldsymbol{n}>\mathbf{= 0}$.

When the appropriate values of $\boldsymbol{m}$ and $\boldsymbol{n}$ are already known for the enhancement of the input image, this method is the fastest one [1]. If the values of $\boldsymbol{m}$ and $\boldsymbol{n}$ are to be guessed, it may try for many times for the proper values of $\boldsymbol{m}$ and $\boldsymbol{n}$. For determining the appropriate values of $\boldsymbol{m}$ and $\boldsymbol{n}$, the histogram of the relevant image is very helpful. By choosing different values of $\boldsymbol{m}$ and $\boldsymbol{n}$, the user can get various options. The user can fine tune the enhancement of an image with more number of user defined parameters. However, the user's convenience may be less in choosing the desired combination from different combinations of $\boldsymbol{m}$ and $\boldsymbol{n}$.

It is a fact that when there is no user defined parameter, no option is available to the user. When there are two or more user defined parameters, choosing the desired combination of the values of the parameters may lead to user's inconvenience. From the point of view of user's convenience, a semiautomatic, single user defined parameter control is preferable if it is possible.

\section{SEMI-AUTOMATIC GLOBAL CONTRAST ENHANCEMENT (SAGCE)}

A semi-automatic version of global contrast enhancement method can be implemented by increasing or decreasing a contrast value from a threshold value for each of the pixel value of the image as

$$
f_{o}=f_{i}+C *\left(f_{i}-g_{\text {mean }}\right)
$$

where, $f_{o}$ is the output pixel value, $f_{i}$ is the input pixel value, $C$ is the contrast gain factor, $\boldsymbol{g}_{\text {mean }}$ is the global mean of the pixel values of the image and the threshold too. Here, $\boldsymbol{g}_{\text {mean }}$ may be different in values from one image to another image. Now, equation (3) can be improved by bringing the $\boldsymbol{g}_{\text {mean }}$ to a fixed value, 0.5 which is the mid value of the intensity range of an image, $[0,1]$. Thus, equation (3) becomes

$$
\begin{aligned}
& f_{o}=\left(f_{i}-g_{\text {mean }}+0.5\right)+C *\left[\left(f_{i}-g_{\text {mean }}+0.5\right)-0.5\right] \\
& \Rightarrow f_{o}=(1+C) *\left(f_{i}-g_{\text {mean }}\right)+0.5
\end{aligned}
$$

With this equation (4), global mean of the pixel values of the original image is always brought into the value 0.5 automatically after the enhancement. There is not a single problem whether the original $\boldsymbol{g}_{\text {mean }}$ is greater than 0.5 or less than 0.5 , the value of the global mean becomes 0.5 after the process. This is illustrated in Fig. 1. In Fig. 1(a), the $\boldsymbol{g}_{\text {mean }}$ of pixel p1 with value 0.3 and pixel $\mathbf{p 2}$ with value 0.5 is 0.4 , which is the condition for less than 0.5. In Fig. 1(b), the $\boldsymbol{g}_{\text {mean }}$ of pixel p1 with value 0.5 and pixel $\mathbf{p 2}$ with value 0.7 is 0.6 , which is the condition for greater than 0.5 . Here, the value of $\boldsymbol{C}$ is taken as 1 for both of Fig. 1(a) and Fig. 1(b). In both of the cases, the enhanced value of $\boldsymbol{g}_{\text {mean }}$ is 0.5 which is a fixed value, and the enhanced values of p1 and p2 become the corresponding values given by the equation (4) after the process.

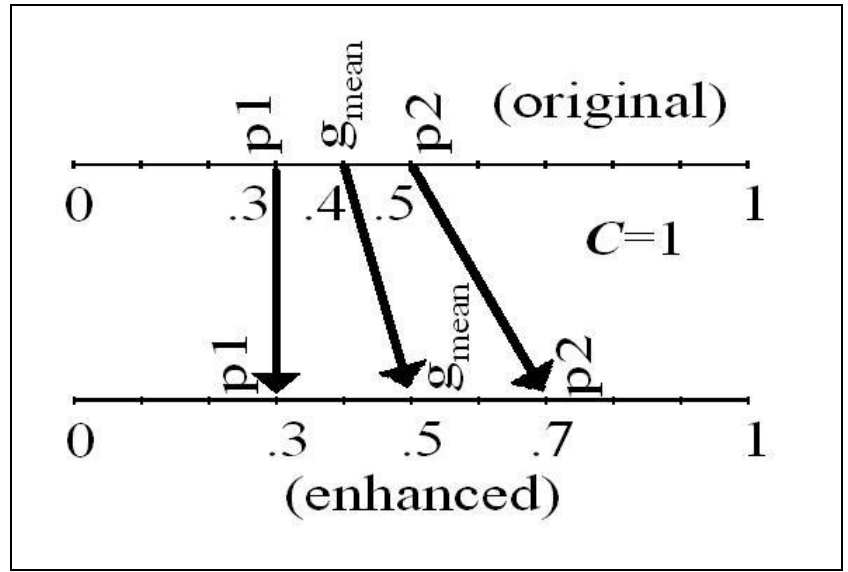

(a) When the original $g_{\text {mean }}$ is less than 0.5

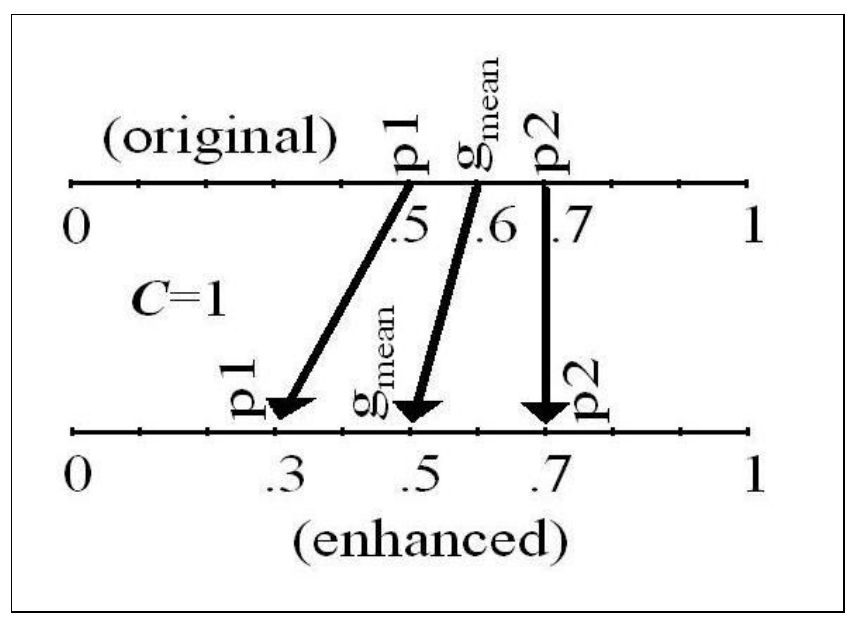

(b) When the original $g_{\text {mean }}$ is greater than 0.5

Fig.1. Illustration of Equation (4)

If the input image is much narrow in the intensity level, the contrast gain factor $\boldsymbol{C}$ is needed to be larger. When the input image is less narrow in the intensity level, $\boldsymbol{C}$ is needed to be smaller. Using only $\boldsymbol{C}$, the user can control the enhancement of the given input image.

\section{EXPERIMENTAL RESULT AND DISCUSSION}

The proposed algorithm has been tested on various input images using MATLAB 7.13.0.564 and the results compared with Global Histogram equalization (GHE) and the method of equation (2) which is controlled by two user defined parameters.

Fig.2 shows the enhanced output images processed with three different methods. Fig.2 (a) is the original image. Fig.2 (b) is the enhanced output image of Fig.2 (a), processed with GHE. Fig.2 (c) is the enhanced output image of Fig.2 (a), processed with equation (2) with $\boldsymbol{m}=1.8$ and $\boldsymbol{n}=0.14$. And Fig.2 (d) is the enhanced output image of Fig.2 (a), processed with SAGCE with $\boldsymbol{C}=0.8$. Here, some of the trees and plants are under exposure for the image processed by GHE and some information is not defined in some walls of the buildings due to over exposure. 


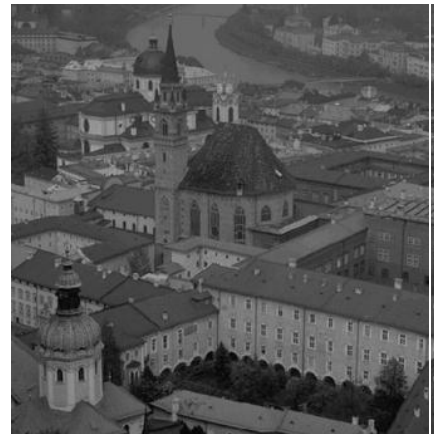

(a) Original

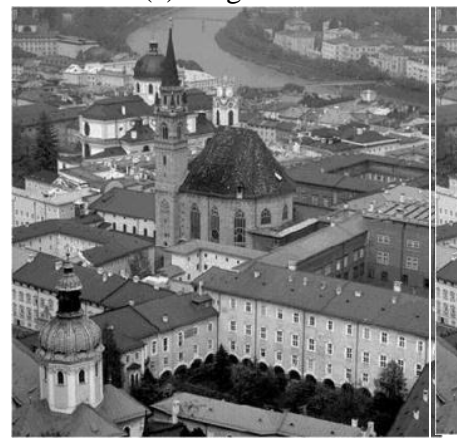

(c) Equation (2) with $\boldsymbol{m}=1.8$, $n=0.14$

Fig.2. Enhancement of the first sample

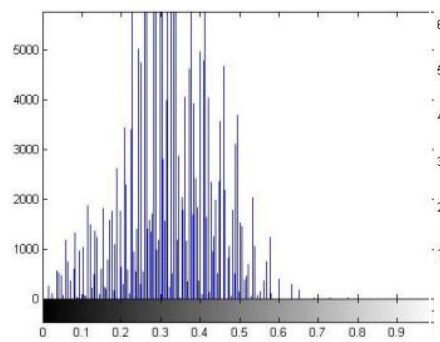

(a) Histogram of 2 (a)

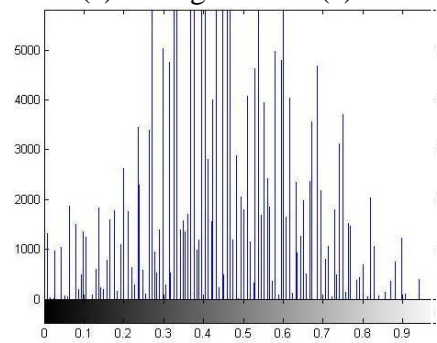

(c) Histogram of 2 (c)

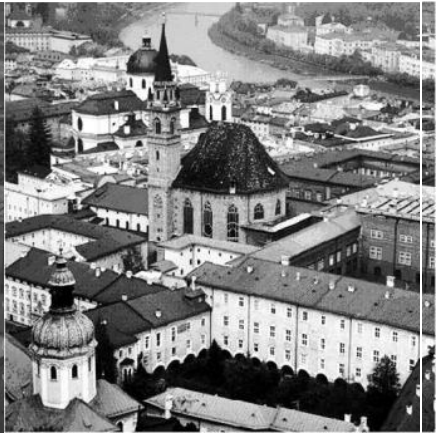

(b) GHE

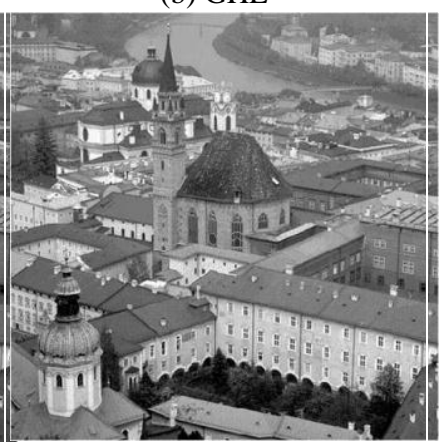

(d) SAGCE with $C=0.8$

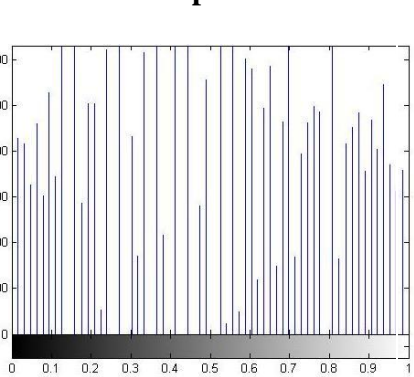

(b) Histogram of 2 (b)

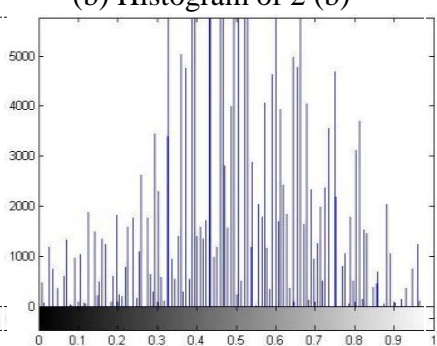

(d) Histogram of 2 (d)

Fig.3. Histograms of fig. 2(a) -2 (d)

Fig.3 shows the histograms of the images shown in Fig.2. Here, Fig.3 (a), Fig.3 (b), Fig.3 (c) and Fig.3 (d) are the histograms of Fig.2 (a), Fig.2 (b), Fig.2 (c) and Fig.2 (d) respectively. Fig.3 (a) is the histogram of the original image. Here, the majority of the pixels reside densely in the left side of the histogram. And the others, Fig.3 (b) - Fig.3 (d) are showing the pixels in a distributed form as the corresponding methods.

Some more enhanced output images are shown in Fig.4 for another sample image. Fig.4 (a) is the original image. Fig.4 (b) is the enhanced output image of Fig.4 (a), processed with GHE.

Fig.4 (c) is the enhanced output image of Fig.4 (a), processed with equation (2) with $\boldsymbol{m}=3.0$ and $\boldsymbol{n}=1.35$. And Fig.4 (d) is the enhanced output image of Fig.4 (a), processed with SAGCE with $\boldsymbol{C}=1.5$. Here, it is difficult to identify the working man in the field in the output image processed by GHE due to the over exposure of the enhancement.

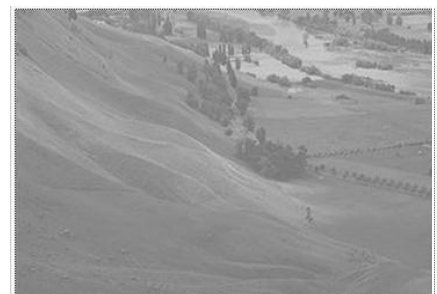

(a) Original

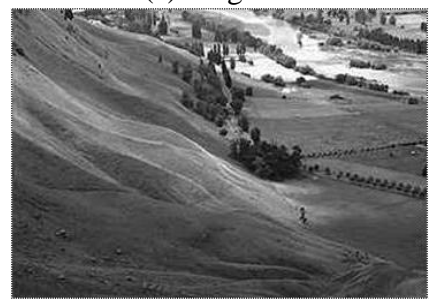

(c) Equation (2) with $\boldsymbol{m}=3.0$, $n=1.35$

Fig.4. Enhancement of the second sample

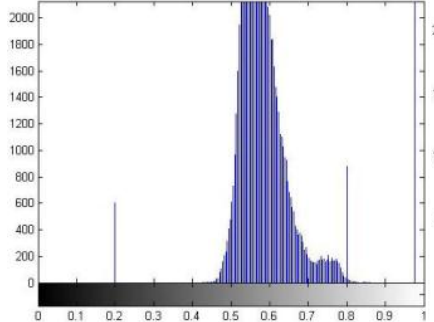

(a) Histogram of 4 (a)

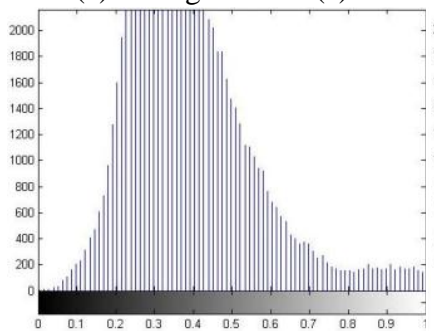

(c) Histogram of 4 (c)

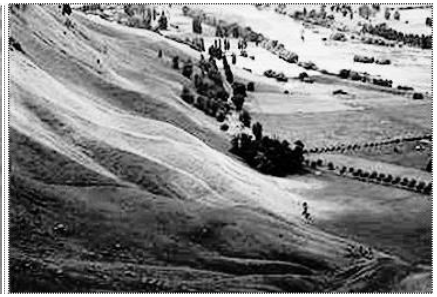

(b) GHE

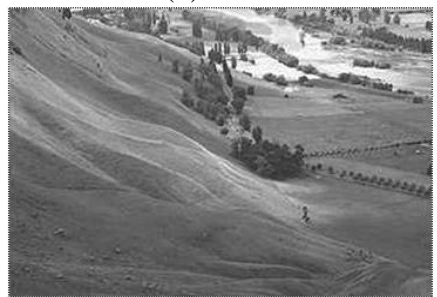

(d) SAGCE with $\boldsymbol{C}=1.5$

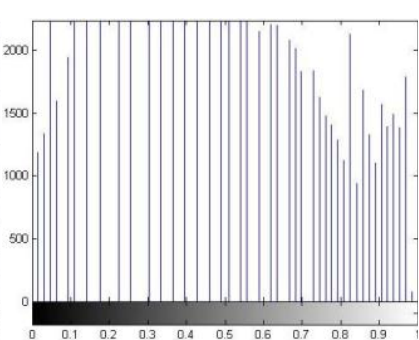

(b) Histogram of 4 (b)

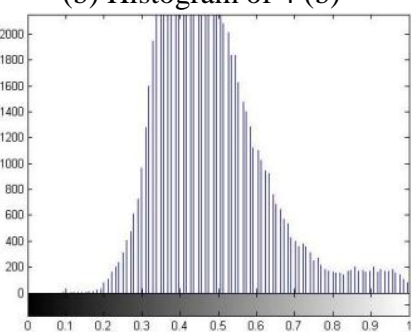

(d) Histogram of 4 (d)
Fig.5. Histograms of fig. 4(a) -4 (d)

Fig.5 shows the histograms of the images shown in Fig.4. Here, Fig.5 (a), Fig.5 (b), Fig.5 (c) and Fig.5 (d) are the histograms of Fig.4 (a), Fig.4 (b), Fig.4 (c) and Fig.4 (d) respectively. Fig.5 (a) is the histogram of the original image. Here, the majority of the pixels reside densely in the right side of the histogram. And the others, Fig.5 (b) - Fig.5 (d) are showing the pixels in a distributed form as the corresponding methods. 


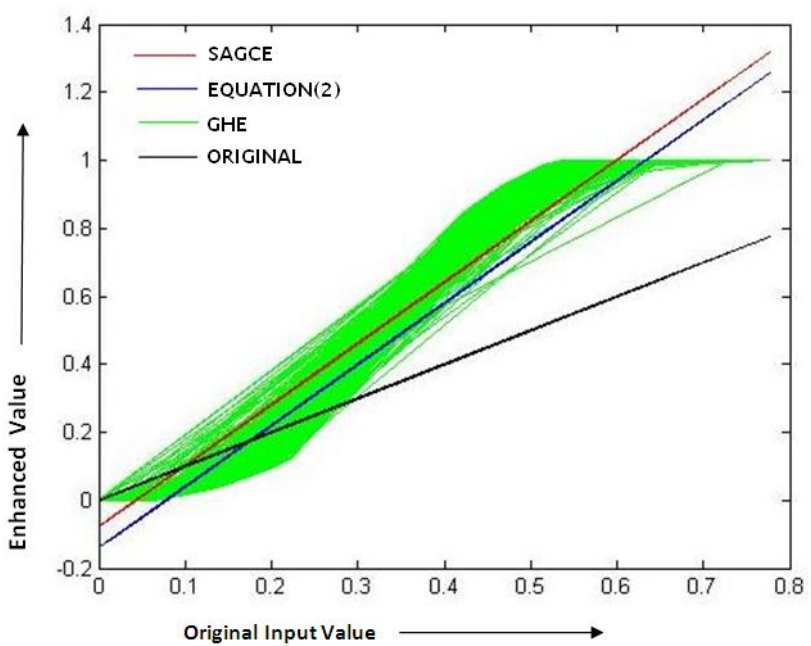

(a) Graph for fig.2.

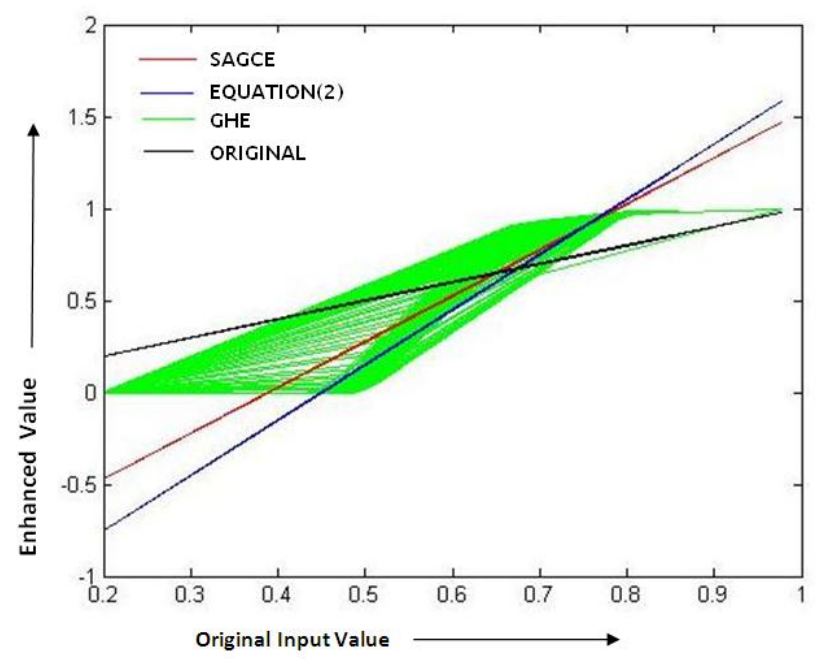

(b) Graph for fig. 4.

Fig. 6 Graphs of Input Values of original pixels against the enhanced output values

In Fig. 6, the graphs of input values of the original pixels against the enhanced output values for each of the methods and the original pixel values itself are shown. Fig. 6(a) represents the graphs for Fig. 2 and Fig. 6(a) represents the graphs for Fig. 4. Here, red colour is represented for SAGCE, blue is represented for equation (2), green is represented for GHE and black is represented for the original pixel values. The enhancement values are shown in the vertical axis and the original values are shown in the horizontal axis. Here, all the graphs except the green one are straight line. This means that for equation (2) and SAGCE methods, the functions used are linear functions and for GHE, the function used is non-linear. By the observations of the enhanced output images, it is found that the output image produced by GHE appears good as a whole image, however, if we look into details of the image, some portions would be found over exposed and some portions would be underexposed in the same image for some images. Although these over exposed and under exposed portions, there is no chance for adjustment of enhancement with GHE because of its automatic system. And using equation (2) or SAGCE, the over exposure and under exposure can be controlled using the user defined parameters. Equation (2) can even control the enhancement more effectively than that of SAGCE. However, using two parameters, $\boldsymbol{m}$ and $\boldsymbol{n}$ in equation (2) is less convenient to the users. From that point of view, SAGCE is much better because of the single user defined parameter $\boldsymbol{C}$ and its semi-automatic nature.

The distinctive features of the proposed method are shown in the comparative statements as shown in the Table 1.

\begin{tabular}{|c|c|c|}
\hline \multicolumn{3}{|c|}{ able 1. A Comparative Statement } \\
\hline GHE & Equation (2) & SAGCE \\
\hline $\begin{array}{l}\text { The system of } \\
\text { processing is } \\
\text { automatic. }\end{array}$ & $\begin{array}{l}\text { The system of } \\
\text { processing is } \\
\text { simple and } \\
\text { manual. }\end{array}$ & $\begin{array}{l}\text { The system of } \\
\text { processing is semi- } \\
\text { automatic. }\end{array}$ \\
\hline $\begin{array}{l}\text { No control option is } \\
\text { available. }\end{array}$ & $\begin{array}{l}\text { It is controlled } \\
\text { with two user's } \\
\text { defined } \\
\text { parameters, and it } \\
\text { is having several } \\
\text { options. }\end{array}$ & $\begin{array}{l}\text { It is controlled with } \\
\text { single user's } \\
\text { defined parameters. }\end{array}$ \\
\hline $\begin{array}{l}\text { The function used } \\
\text { is a non-linear } \\
\text { function. }\end{array}$ & $\begin{array}{l}\text { The function } \\
\text { used is a linear } \\
\text { function. }\end{array}$ & $\begin{array}{l}\text { The function used } \\
\text { is a linear function. }\end{array}$ \\
\hline $\begin{array}{l}\text { Due to the } \\
\text { automatic nature of } \\
\text { the processing } \\
\text { system, it is the } \\
\text { most user } \\
\text { convenient method, } \\
\text { however, some } \\
\text { portions of the } \\
\text { output image is } \\
\text { over exposed or } \\
\text { under exposed for } \\
\text { some kind of } \\
\text { images, and it is not } \\
\text { adjustable. }\end{array}$ & $\begin{array}{l}\text { It is having more } \\
\text { adjustable facility } \\
\text { in the } \\
\text { enhancement of } \\
\text { the input image } \\
\text { because of two } \\
\text { user's defined } \\
\text { parameters. } \\
\text { However, there } \\
\text { may be less in } \\
\text { user- } \\
\text { convenience. }\end{array}$ & $\begin{array}{l}\text { This system is more } \\
\text { user convenient } \\
\text { than that of } \\
\text { equation (2) } \\
\text { because of its semi- } \\
\text { automatic nature, } \\
\text { and the } \\
\text { enhancement of the } \\
\text { input image too can } \\
\text { be adjustable with } \\
\text { the single user- } \\
\text { defined parameter. }\end{array}$ \\
\hline
\end{tabular}

\section{CONCLUSION}

Local Contrast Enhancement is limited to global contrast for some images. For the need of global contrast, many global contrast enhancement methods have been utilized. This paper presents the implementation of a semi-automatic global contrast enhancement using a single user defined parameter, which is very convenient to the user. This semi-automatic global contrast enhancement method will be a good step for the combination with other local contrast enhancement methods because some of the global contrast enhancement methods cannot be combined with other local contrast enhancement methods. The combination of this semi-automatic global contrast enhancement method and other local contrast enhancement method is the subject of future interest.

\section{REFERENCES}

[1] S. Somorjeet Singh, H. Mamata Devi, Th. Tangkeshwar Singh, O. Imocha Singh, "A New Easy Method of Enhancement of Low Contrast Image Using Spatial Domain," International Journal of Computer Applications (0975 - 8887), February 2012, Volume 40- No.1.

[2] R. C. Gonzalez and R. E. Woods, 2008, Digital Image Processing, $3^{\text {rd }}$ edition, Prentice Hall

[3] Sascha D. Cvetkovic and Peter H. N. de With, "Image enhancement circuit using non-linear processing curve and constrained histogram range equalization," Proc. of SPIEIS\&T Electronic Imaging, 2004, Vol. 5308, pp. 11061116. 
[4] M.A. Yousuf, M.R.H. Rakib, "An Effective Image Contrast Enhancement Method Using Global Histogram Equalization," Journal of Scientific Research, 2011, Vol. 3, No. 1.

[5] I. Altas, J. Louis, and J. Belward, "A variational approach to the radiometric enhancement of digital imagery," IEEE Trans. Image Processing, June 1995, vol. 4, pp. 845-849.

[6] L. Dorst, "A local contrast enhancement filter," In Proc. 6th Int. Conference on Pattern recognition, 1982, pages 604-606, Munich, Germany.

[7] D. Mukherjee and B. N. Chatterji, "Adaptive neighborhood extended contrast enhancement and its modification," Graphical Models and Image Processing, 1995, 57:254-265.

[8] R. B. Paranjape, W. M. Morrow, and R. M. Rangayyan, "Adaptive-neighborhood histogram equalization for image enhancement," Graphical Models and Image Processing, 1992, 54:259-267.

[9] S. M. Pizer, E. P. Amburn, J. D. Austin, R. Cromartie, A. Geselowitz, T. Geer, B. H. Romeny, J. B. Zimmerman, and K. Zuiderveld, "Adaptive histogram modification and its variation," Computer Vision, Graphics and Image Processing, 1987, 39:355-368.

[10] J. S. Lee, "Digital image enhancement and noise filtering by use of local statistics," IEEE Trans. on Pattern Analysis and Machine Intelligence, 1980, PAMI-2:165-
[11] J. S. Lee, "Refined filtering of image noise using local statistics," Computer Graphics and Image Processing, 1981, 15:380-.

[12] P.M. Narendra and R.C. Fitch, "Real-time adaptive contrast enhancement," IEEE Trans. PAMI, Vol.3, no. 6, pp.655-661, 1981.

[13] D.-C. Chang and W.-R. Wu, "Image contrast enhancement based on a histogram transformation of local standard deviation," IEEE Trans. MI, Aug. 1998, vol. 17, no. 4, pp. 518-531.

[14] K. Schutte, "Multi-Scale Adaptive Gain Control of IR Images," Infrared Technology and Applications XXIII, Proceedings of SPIE, 1997, Vol. 3061, pp.906-914.

[15] Sascha D. Cvetkovic, Johan Schirris and Peter H. N. de With, "Locally-Adaptive Image Contrast Enhancement Without Noise And Ringing Artifacts," IEEE, ICIP 2007.

[16] S. Somorjeet Singh, Th. Tangkeshwar Singh, H. Mamata Devi, Tejmani Sinam, "Local Contrast Enhancement Using Local Stan dard Deviation," International Journal of Computer Applications (0975-888), June 2012, Volume 47- No.15.

[17] T.-L. Ji, M. K. Sundareshan, and H. Roehrig, "Adaptive image contrast enhancement based on human visual properties," IEEE trans. Med. Imag., Aug. 1994, vol. 13, pp. 573-586 